\title{
Aspectos Produtivos da Raça Pardo-Suíça no Brasil. Fatores de Ajustamento, Produção de Leite e de Gordura, e Parâmetros Genéticos ${ }^{1}$
}

\author{
Francisco Palma Rennó ${ }^{2}$, José Carlos Pereira ${ }^{3}$, Cláudio Vieira de Araújo ${ }^{4}$, \\ Robledo de Almeida Torres ${ }^{3}$, Marcelo Teixeira Rodrigues ${ }^{3}$, Luciana Navajas Rennó ${ }^{5}$, \\ Rita Flávia Miranda de Oliveira ${ }^{3}$, Fernando da Rocha Kaiser ${ }^{6}$
}

\begin{abstract}
RESUMO - Foi realizado um estudo sobre o desempenho produtivo da raça Pardo-Suíça com o objetivo de estimar fatores de ajustamento, avaliar fatores de ambiente e genéticos que influenciam a produção de leite, de gordura e a porcentagem de gordura, e estimar parâmetros genéticos para estas características produtivas. Foram avaliadas 11189 lactações de 5382 vacas Pardo-Suíças, de 1980 a 1999 , oriundas de 201 rebanhos, sendo os registros de produção do serviço de controle leiteiro realizado pela Associação Brasileira de Criadores de Gado Pardo-Suíço. As lactações foram ajustadas por meio de fatores multiplicativos de ajustamento para duas ordenhas, períodos de lactação de 305 dias e produção a idade adulta. As médias estimadas, os respectivos desvios-padrão e os coeficientes de variação da produção de leite, produção de gordura e percentagem de gordura foram 5791,50 $\pm 1211,58 \mathrm{~kg}$ e 20,92\%; $217,25 \pm 47,36 \mathrm{~kg}$ e $21,80 \%$ e 3,78 $\pm 0,34$ e 9,16\%, respectivamente. Os efeitos de ano e época de partos, interação ano-época de partos, rebanho e grupo genético influenciaram as características estudadas, com exceção da época de partos sobre a percentagem de gordura. Os coeficientes de herdabilidade e repetibilidade estimados para a produção de leite e gordura foram 0,37 e 0,40 , e 0,36 e 0,37 , respectivamente. A correlação genética entre a produção de leite e de gordura encontrada neste estudo foi de 0,96 . Os resultados obtidos revelam a necessidade do ajustamento das produções de leite e gordura para os efeitos avaliados. As médias de produção de leite, de gordura e à percentagem de gordura apresentada demonstram o elevado desempenho produtivo da raça Pardo-Suíça nos rebanhos brasileiros.
\end{abstract}

Palavras-chave: características produtivas, fatores de ajustamento, Pardo-Suíço, parâmetros genéticos

\section{Productive Aspects of the Brown Swiss Breed in Brazil1: Adjustment Factors, Milk and Fat Yields, and Genetic Parameters}

\begin{abstract}
A study was carried on performance of the Brown Swiss cattle with the objective of estimating adjustment factors, evaluate some environment and genetics factors that affect milk and fat yields and fat percentage, and estimate genetic parameters for this productive traits. It was appraised 11,189 lactations out of 5,382 Brown Swiss cows, from 1980 to 1999, originated from 201 herds, provided by milking record service accomplished by the Brown Swiss Cattle Breeder's Association of Brazil. Productions were adjusted by a multiplicative factors for two milkings, 305-day and production at the mature age. The estimated averages and its standard deviation and the coefficient of variation for milk and fat yield and fat percentage were, respectively, 5,791.50 $\pm 1,211.58 \mathrm{~kg}$ and $20.92 \% ; 217.25$ $\pm 47.36 \mathrm{~kg}$ and $21.80 \%$ and $3.78 \pm 0.34$ and $9.16 \%$, respectively. The effects of year and season of calving, year $\mathrm{x}$ season of calving interaction, herd and genetic group were significant, except for season of calving on fat percentage. Heritability estimates and repeatability for milk and fat yields were 0.37 and 0.40 , and 0.36 and 0.37 respectively. Genetic correlation between milk and fat yield was 0.96 . The obtained results showed the need to adjustment milk and fat yields for two milkings, 305-day and production at the mature age. It was concluded that the Brown Swiss breed has a great potential for production in Brazilian dairy herds.
\end{abstract}

Key Words: adjustment factors, Brown Swiss, genetic parameters, productive characteristics

\section{Introdução}

O conhecimento do potencial produtivo de animais destinados a produção leiteira e dos fatores que interferem na expressão deste potencial são primordiais para que sejam delineados os objetivos finais daquela exploração pecuária e ainda, quais atitudes deverão ser tomadas para a plena produção dos animais selecionados para determinado sistema de produção.

Uma característica peculiar da pecuária brasileira, principalmente a leiteira, é a grande variabilidade de

\footnotetext{
1 Parte da Dissertação apresentada a UFV, para obtenção do título de "Magister Scientiae".

2 Doutorando do Departamento de Zootecnia/UFV, Bolsista do CNPq. E.mail: fprenno@alunos.ufv.br

3 Professor do Departamento de Zootecnia/UFV, Bolsista do CNPQ. E.mail: jcarlos@ufv.br

${ }^{4}$ Professor da Faculdade de Ciências Agrárias do Pará - FCAP. E.mail: araujocv@bol.com.br

5 Professora da Universidade Estadual do Oeste do Paraná - UNIOESTE, Marechal Cândido Rondon

${ }^{6}$ Superintendente Técnico da Associação Brasileira dos Criadores de Gado Pardo-Suíço.
} 
sistemas de produção. Isto torna ainda mais difícil a correta escolha de determinada raça ou cruzamento de bovinos para estes rebanhos. Desta forma, o conhecimento do potencial produtivo de cada raça e de cada cruzamento utilizado nas condições do país deve ser estudado para que se tenha segurança quando da indicação de determinado animal para os diversos sistemas de produção.

No Brasil, os sistemas de produção de leite são baseados, em sua maioria, em animais da raça Holandesa e de seus cruzamentos com outras raças, principalmente as de origem zebuína, como o Gir Leiteiro e o Guzerá. Entretanto, nos últimos anos, outras raças de origem européia utilizadas para a produção de leite têm aumentado sua população, tanto de animais puros de origem como de seus cruzamentos. Dentre estas, a raça Pardo-Suíça tem atendido as exigências produtivas de variados tipos de sistemas de produção, demonstrando grande crescimento, especialmente durante a última década (Rennó, 2000).

Segundo Rennó (2000), atualmente temos no país cerca de 66.000 animais da raça Pardo-Suíça registrados, distribuídos em 23 estados da federação, existindo atualmente apenas 3 estados brasileiros sem a presença de criadores. Kaiser (1998) cita que, quando são contados animais sem registro e com participação de sangue em animais cruzados, o número de animais desta raça é de, aproximadamente, 1 milhão de cabeças.

No Brasil são poucos os trabalhos de pesquisa em bovinocultura leiteira que tem avaliado o potencial produtivo de bovinos, especialmente com animais da raça Pardo-Suíça. Assim, a avaliação do potencial produtivo do Pardo-Suíço em sistemas de produção utilizados no Brasil, em condições intertropicais, possibilita a obtenção de resultados que nos demonstram o potencial produtivo destes animais nesta particular situação de manejo e ambiente.

As variações nas características produtivas em bovinos leiteiros são atribuídas a diferenças de fatores de meio ambiente (não-genéticos) e a diferenças de herança (genéticos). Muitos efeitos de ambiente têm sido citados na literatura como causas significativas de variações nas características produtivas. Dentre os fatores de maior impacto na produção de leite de bovinos, citam-se: ano e estação de parto, rebanho, grupo genético, número de ordenhas, período de lactação e idade ou ordem de partos.

O efeito do ano de parto sobre a produção de leite, gordura e a porcentagem de gordura reflete um complexo de influências que podem ser de ordem climática, de manejo, alimentação, rentabilidade da atividade leiteira e a alterações genéticas provocadas pela seleção. Almeida et al. (1999) e Ribas et al. (1996), trabalhando com lactações de vacas das raças Pardo-Suíça e Holandesa, respectivamente, verificaram que o ano de parto influenciou a produção de leite, de gordura e porcentagem de gordura.

De forma semelhante, a época de parto influencia diretamente a produção de vacas leiteiras, sendo relacionada a diferenças qualitativa e quantitativa de alimentos, principalmente de forragens, e ainda climáticas, entre as várias épocas do ano (Freeman, 1973). Pimpão et al. (1997) e Ray et al. (1992), ambos trabalhando com dados de produção de animais da raça Holandesa, verificaram que a época de partos influencia as produções de leite e de gordura.

Os rebanhos afetam de forma diferenciada as características produtivas. Este efeito pode ser explicado devido a variações de manejo, de alimentação, de localização, de composição genética de sua população e de critérios de seleção. Desta forma, McDowell et al. (1976) e Conceição Jr. et al. (1993) citam que a maior parte da variação na produção de leite e de gordura é devido à influência do rebanho em que o animal é criado.

Uma importante variação nas características produtivas se refere à raça, grupo genético e/ou grau de sangue dos animais estudados. Segundo Campos (1987), os grupos genéticos que formam um rebanho em determinado período deverão alterar a produção por vaca em lactação, já que cada raça e/ou cruzamento possuem diferentes capacidades de adaptação às condições de meio ambiente.

A comparação de animais destinados à produção de leite e gordura exige que as produções destes estejam ajustadas a fatores não genéticos para uma avaliação correta dos indivíduos. Dentre estes efeitos, o número de ordenhas diárias, a duração da lactação e a idade da vaca ao parto exercem influência sobre a produção de vacas leiteiras e necessitam ser corrigidos antes da predição do mérito genético e/ou do potencial produtivo de cada animal.

O número de ordenhas diárias influência a produção de leite, onde vacas ordenhadas três vezes ao dia apresentam aumentos de 5 a $20 \%$ quando comparadas com animais ordenhados duas vezes ao dia (Amos et al., 1985; DePeters et al., 1985), além de apresentar conteúdo de gordura mais elevado quando as vacas são ordenhadas mais freqüentemente. Segun-

R. Bras. Zootec., v.31, n.5, p.2043-2054, 2002 
do Ribas (1981), a duração da lactação é fator responsável por considerável variação das produções de leite e gordura. Barbosa et al. (1994) citam como período de lactação ideal o de aproximadamente dez meses, o que possibilitaria a ocorrência de um parto por ano e maiores produções de leite por vaca durante sua vida produtiva.

Martinez et al. (1990) concluíram ser necessário o uso de fatores de ajuste para a idade ao parto. McDowell et al. (1976), ao avaliarem o desempenho produtivo de vacas da raça Holandesa no México, concluíram que à máxima produção de leite era alcançada com vacas na idade adulta, sendo a produção destas aproximadamente $18 \%$ superiores a produções de vacas de primeira lactação.

A avaliação dos parâmetros genéticos das características produtivas são importantes para o pleno progresso produtivo de rebanhos e empresas rurais. Estes conceitos dão estimativas da magnitude da influência dos fatores ambientais (não genéticos) e dos fatores genéticos. Assim, a avaliação da herdabilidade, repetibilidade e correlações genéticas da produção de leite e de gordura são importantes na implementação de programas de seleção de bovinos leiteiros (Coelho, 1990; Balieiro, 1997; Freitas et al., 1999a).

O objetivo deste estudo foi avaliar o desempenho produtivo de animais da raça Pardo-Suíça, estimar fatores de ajustamento e analisar fatores de meio ambiente e genéticos que influenciam a produção de leite, a produção de gordura e a porcentagem de gordura, e estimar parâmetros genéticos para o rebanho Pardo-Suíço do Brasil.

\section{Material e Métodos}

Os dados utilizados neste estudo são provenientes do Controle Leiteiro da Associação Brasileira dos Criadores de Gado Pardo-Suíço (ABCGPS), sendo fornecidos através de convênio mantido entre o Departamento de Zootecnia da Universidade Federal de Viçosa e a ABCGPS. Os registros de produção utilizados foram constituídos de 11189 lactações de 5382 vacas distribuídas em 201 rebanhos, com datas de partos nos anos de 1980 a 1999.

Os dados de produção foram editados para eliminação de registos de produção e ascendentes incompletos, lactações inferiores a 150 e superiores à 365 dias, registros de produção inferiores a 1000 ou superiores a $15000 \mathrm{~kg}$ de leite. Animais com idade ao parto menor que 18 meses e maior que 197 meses

R. Bras. Zootec., v.31, n.5, p.2043-2054, 2002 também foram eliminados.

Os meses de parto das vacas foram agrupados em quatro épocas: época 1, que corresponde aos meses de janeiro a março; época 2, que corresponde aos meses de abril a junho; época 3, correspondendo aos meses de julho a setembro; e época 4, que corresponde aos meses de outubro a dezembro. As épocas 1 e 4 correspondem aos meses de maior incidência de chuvas e maior temperatura e umidade relativa do ar, enquanto que as épocas 2 e 3 correspondem aos meses de menor incidência de chuvas e menores temperaturas e umidade relativa do ar, já que a maior parte dos dados corresponde a rebanhos localizados nas regiões sudeste e sul do país.

Os rebanhos constituintes do arquivo analisado foram editados de forma que cada rebanho possuísse, no mínimo, três lactações por ano. De acordo com a informação de ascendência e a origem dos animais, as vacas foram classificadas em animais do grupo genético Pardo-Suíço puro de origem (PO), puro por cruzamento (PC) e puro de origem importada (POI) (animais nascidos fora do Brasil e importados).

Na avaliação dos parâmetros genéticos exigiu-se que cada reprodutor possuísse filhas em pelo menos dois diferentes rebanhos, e ainda, que cada rebanho pertencente a este arquivo tivesse pelo menos dois animais em produção dentro de cada ano. Desta forma, para a avaliação dos coeficientes de herdabilidade, repetibilidade e correlações genéticas da produção de leite e gordura foram utilizadas 4205 lactações, de 2028 vacas, filhas de 239 reprodutores e oriundas de 196 rebanhos, com datas de partos entre os anos de 1980 e 1999.

As lactações foram ajustadas previamente para o número de ordenhas diárias (duas ordenhas $-2 \mathrm{x}$ ), duração da lactação (305 dias) e para a produção na idade adulta da vaca. Para a obtenção destes fatores de ajustamento foi verificado que o efeito dos fatores citados anteriormente foram significativos sobre as características produtivas estudadas, sendo utilizado o seguinte modelo estatístico:

$\hat{\mathrm{Y}}_{i j k l m n o p}=\mu+a_{i}+e_{j}+a e_{(i j)}+r_{k}+g_{i}+g_{l}+o_{m}+d_{n}+p_{o}+\varepsilon_{i j k l m n o i}(1)$ em que: $\hat{\mathrm{Y}}_{i j k l m n o p}$ é a variável resposta na lactação p; no i-ésimo ano de parto; na j-ésima época de parto, no k-ésimo rebanho, do l-ésimo grupo genético, na mésima frequência de ordenha; na n-ésima duração de lactação e o-ésima idade da vaca ao parto; $\mu$, a média geral da característica estudada; $a_{i}$, o efeito fixo do ano de parto $\mathrm{i} ; e_{j}$, efeito fixo da época de parto $\mathrm{j} ; a e_{(i j)}$, a interação entre o efeito ano x época de parto; $r_{k}$, 
efeito fixo do rebanho $\mathrm{k} ; g_{l}$, efeito fixo do grupo genéticoj; om, efeito fixo de número de ordenhas; $d_{n}$, efeito fixo da classe de duração de lactação $1 ; p_{o}$, efeito fixo da classe de idade da vaca no parto k; $\varepsilon_{i j k l m n o p}$, efeito aleatório residual associado a cada observação, com média 0 e variância .

O ajustamento da produção de leite e de gordura de três para duas ordenhas diárias foi efetuado utilizando-se as fórmulas apresentadas por Wiggans e Dickinson (1985) citados em Houri Neto (1998), resultando em fatores de ajustamento multiplicativos para o número de ordenhas diárias. A duração da lactação foi agrupada em classes para serem utilizadas nas estimativas dos fatores de ajustamento. Os registros da produção total de leite, gordura e percentagem de gordura foram corrigidos para 305 dias de lactação por meio de fatores multiplicativos de ajustamento, obtidos a partir das estimativas das classes de duração de lactação por meio de um modelo estatístico similar ao modelo 1 , com a exclusão da variável número de ordenhas.

Foi estimada uma equação de regressão em função da idade média de cada classe de idade da vaca ao parto, sendo obtida as idades de máxima produção de leite e gordura para a realização do ajuste para aquelas idades. Posteriormente, foram obtidos os fatores multiplicativos de ajustamento a partir das médias de quadrados mínimos da produção de leite e gordura de acordo com as classes de idade da vaca ao parto, sendo utilizado um modelo estatístico similar ao modelo 1 , com a exclusão das variáveis número de ordenhas e período de lactação.

Para a análise dos efeitos de meio ambiente e genéticos sobre as produções de leite, de gordura e percentagem de gordura, foi empregado o método dos quadrados mínimos, utilizando o procedimento GLM do SAS (SAS,1991). Após a correção das características produtivas para o número de ordenhas diárias, período de lactação e idade da vaca ao parto, foram realizados estudos dos efeitos de meio ambiente e genético sobre as características produtivas, sendo utilizando um modelo estatístico semelhante ao modelo 1 com a exclusão das variáveis número de ordenhas, período de lactação e idade da vaca ao parto.

As estimativas de componentes de (co)variâncias e dos parâmetros genéticos foram obtidas do programa MTDFREML (Multiple Trait Derivative Free Restricted Maximum Likelihood), descrito por Boldman et al. (1995), utilizando a metodologia de máxima verossimilhança restrita livre de derivadas. Foram considerados os efeitos fixos de rebanho, ano e época de partos, e grupo genético. O critério adotado na convergência da variância dos valores do simplex foi de, no mínimo, $10^{-9}$. Adotou-se o seguinte modelo:

$$
\begin{gathered}
\mathrm{y}=\mathrm{X} \beta \underset{\sim}{\beta}+\mathrm{Z}_{\mathrm{a}} \underset{\sim}{\mathrm{a}}+\mathrm{W}_{\mathrm{p}} \underset{\sim}{\mathrm{p}}+\underset{\sim}{\varepsilon} \\
{\left[\begin{array}{c}
\mathrm{y}_{1} \\
\mathrm{y}_{2}
\end{array}\right]=\left[\begin{array}{cc}
\mathrm{X}_{1} & 0 \\
0 & \mathrm{X}_{2}
\end{array}\right]\left[\begin{array}{c}
\beta_{1} \\
\tilde{\beta_{2}}
\end{array}\right]+\left[\begin{array}{cc}
\mathrm{Z}_{1} & 0 \\
0 & \mathrm{Z}_{2}
\end{array}\right]\left[\begin{array}{c}
\mathrm{a}_{1} \\
\tilde{\mathrm{a}_{2}} \\
\sim
\end{array}\right]+\left[\begin{array}{cc}
\mathrm{W}_{1} & 0 \\
0 & \mathrm{~W}_{2}
\end{array}\right]\left[\begin{array}{c}
\mathrm{p}_{1} \\
\tilde{\mathrm{p}_{2}}
\end{array}\right]+\left[\begin{array}{c}
\varepsilon_{1} \\
\tilde{\varepsilon_{2}} \\
\sim
\end{array}\right],}
\end{gathered}
$$

em que: $\mathrm{y}_{1}$ é um vetor de observações para a característica i; $\left[\mathrm{X}_{1}\right.$ é uma matriz de incidência relativa aos registros de produção da característica i para os efeitos fixos; $\beta_{1}$ é um vetor dos efeitos fixos para a característica i; $Z_{1}$ é uma matriz de incidência relativa aos registros de produção da característica i para $o$ efeito de animal; ${ }_{\sim}^{a_{1}}$ é um vetor de efeitos aleatórios de animal para a característica $i ; W_{1}$ é uma matriz de incidência relativa aos registros de produção da característica i para os efeitos permanentes de meio ambiente; $\mathrm{p}_{1}$ é um vetor de efeito permanente de meio para a característica $\mathrm{i}$; e e é um vetor de erros aleatórios para a característica i.

\section{Resultados e Discussão}

\section{Fatores de Ajustamento}

O ajustamento das produções de leite e de gordura de três para duas ordenhas diárias resultou em fatores de ajustamento multiplicativos de 0,81 para produção de leite e de 0,85 para produção de gordura, sendo semelhantes aos verificados por Amos et al. (1985) e DePeters et al. (1985). As médias estimadas e respectivos desvios-padrão utilizados para a estimativa dos fatores de ajustamento para número de ordenhas podem ser observadas na Tabela 1 .

Os fatores de ajustamento para as produções de leite, de gordura e para a percentagem de gordura, obtidos em cada classe de duração de lactação, podem ser encontrados na Tabela 2. Os valores dos fatores multiplicativos para o período de lactação estimados foram próximos aos encontrados por Torres (1998), Costa (1998) e Araújo (2000).

As idades encontradas de maiores produções de leite e gordura, respectivamente, foram de 72,05 e 68,74 meses de idade, enquanto que a idade encontrada de menor percentagem de gordura foi 120,55

R. Bras. Zootec., v.31, n.5, p.2043-2054, 2002 
Tabela 1 - Número de observações (N), médias estimadas e desvios-padrão (DP) das produções de leite e de gordura, de acordo com o número de ordenhas

Table 1 - Number of observations (N), estimate averages and standard-deviation (DP) of the productions of milk and fat, in agreement with the number of milking daily

\begin{tabular}{|c|c|c|c|c|c|}
\hline \multirow[t]{2}{*}{$\begin{array}{l}\text { Variável } \\
\text { Variable }\end{array}$} & \multirow[t]{2}{*}{$\begin{array}{l}\mathrm{N} \\
N\end{array}$} & \multicolumn{2}{|c|}{$\begin{array}{l}\text { Produção de leite } \\
\text { Milk production }\end{array}$} & \multicolumn{2}{|c|}{$\begin{array}{l}\text { Produção de gordura } \\
\text { Fat production }\end{array}$} \\
\hline & & $\begin{array}{c}\text { Médias } \\
\text { Averages }\end{array}$ & $\begin{array}{c}\mathrm{DP} \\
\text { Standard deviation } \\
\end{array}$ & $\begin{array}{c}\text { Médias } \\
\text { Averages }\end{array}$ & $\begin{array}{c}\mathrm{DP} \\
\text { Standard deviation } \\
\end{array}$ \\
\hline $\begin{array}{l}\text { Duas ordenhas } \\
\text { Two milking }\end{array}$ & 9317 & 5267,68 & 1982,81 & 198,99 & 75,17 \\
\hline $\begin{array}{l}\text { Três ordenhas } \\
\text { Three milking }\end{array}$ & 1876 & 6491,37 & 2092,46 & 233,50 & 75,10 \\
\hline
\end{tabular}

Tabela 2 - Fatores de correção multiplicativos da produção de leite, gordura e percentagem de gordura (2x), para um período de lactação de 305 dias

Table 2 - Multiplicative adjustment factors of the production of milk, fat and fat percentage (2x), for a period of lactation of 305 days

\begin{tabular}{|c|c|c|c|}
\hline \multicolumn{2}{|c|}{$\begin{array}{l}\text { Período de lactação (dias) } \\
\text { Lactation period (days) }\end{array}$} & \multicolumn{2}{|l|}{$\begin{array}{l}\text { Fatores de correção } \\
\text { Adjustment factors }\end{array}$} \\
\hline & $\begin{array}{l}\text { Produção de leite } \\
\text { Milk production }\end{array}$ & $\begin{array}{l}\text { Produção de gordura } \\
\text { Fat production }\end{array}$ & $\begin{array}{c}\text { Percentagem de gordura } \\
\text { Fatpercentage }\end{array}$ \\
\hline $150-169$ & 2,207206 & 2,241354 & 1.024866 \\
\hline $170-179$ & 1,892385 & 1,867899 & 1,004366 \\
\hline $180-189$ & 1,832614 & 1,839322 & 1,028555 \\
\hline 190-199 & 1,798827 & 1,811509 & 1,001037 \\
\hline 200-209 & 1,737584 & 1,722864 & 1,005364 \\
\hline $210-219$ & 1,608742 & 1,602357 & 1,005221 \\
\hline $220-229$ & 1,483849 & 1,469939 & 1,001090 \\
\hline $230-239$ & 1,470447 & 1,474424 & 1,013043 \\
\hline $240-249$ & 1,395457 & 1,381679 & 1,000320 \\
\hline $250-259$ & 1,305680 & 1,290417 & 0,994399 \\
\hline $260-269$ & 1,218843 & 1,210858 & 1,000231 \\
\hline $270-279$ & 1,168336 & 1,172869 & 1,004474 \\
\hline $280-289$ & 1,117609 & 1,109862 & 0,996853 \\
\hline $290-299$ & 1,051954 & 1,053261 & 0,999678 \\
\hline $300-309$ & 1 & 1 & 1 \\
\hline $310-319$ & 0,975020 & 0,973694 & 0,996072 \\
\hline $320-329$ & 0,951243 & 0,947880 & 0,992678 \\
\hline $330-339$ & 0,909267 & 0,907414 & 0,993809 \\
\hline $340-349$ & 0,887753 & 0,886165 & 0,994547 \\
\hline $350-364$ & 0,846889 & 0,845128 & 0,994586 \\
\hline 365 & 0,784561 & 0,787525 & 0,995290 \\
\hline
\end{tabular}

meses. As equações de regressão e os respectivos gráficos de produção de leite, de gordura e percentagem de gordura relacionados com a idade de máxima produção podem ser encontrados nas Figura 1, 2 e 3. As estimativas dos fatores de correção para a idade da vaca ao parto foram semelhantes aos valores relatados por Coelho (1990). Por meio das idades de máximas produções foram obtidos os fatores de ajustamento para a idade da vaca ao parto (Tabela 3 ).

As médias estimadas ajustadas, os respecti- vos desvios-padrão e os coeficientes de variação da produção de leite, produção de gordura e percentagem de gordura referentes a 11189 lactações de 5382 vacas da raça Pardo-Suíça, no período de 1980 a 1999 , foram de $5791,50 \pm 1211,58 \mathrm{~kg} \mathrm{e}$ $20,92 \% ; 217,25 \pm 47,36 \mathrm{~kg}$ e $21,80 \%$ e $3,78 \pm 0,34$ e $9,16 \%$. O resumo da análise de variância da produção de leite, gordura, e percentagem de gordura, ajustadas para duas ordenhas $(2 \mathrm{x}), 305$ dias e idade adulta (ME) encontram-se na

R. Bras. Zootec., v.31, n.5, p.2043-2054, 2002 


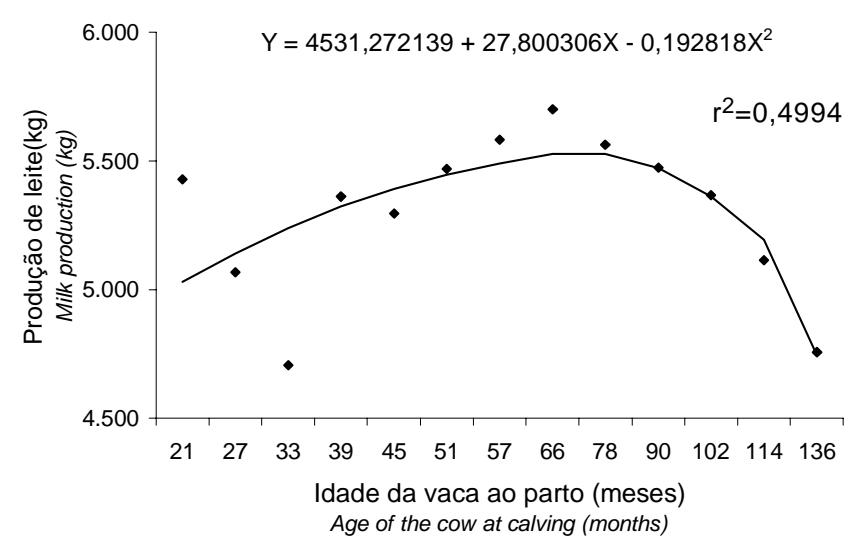

Figura 1 - Produção de leite $(\mathrm{kg})$, segundo a idade da vaca ao parto.

Figure 1 - Milk production (kg), according to the age of the cow at calving.

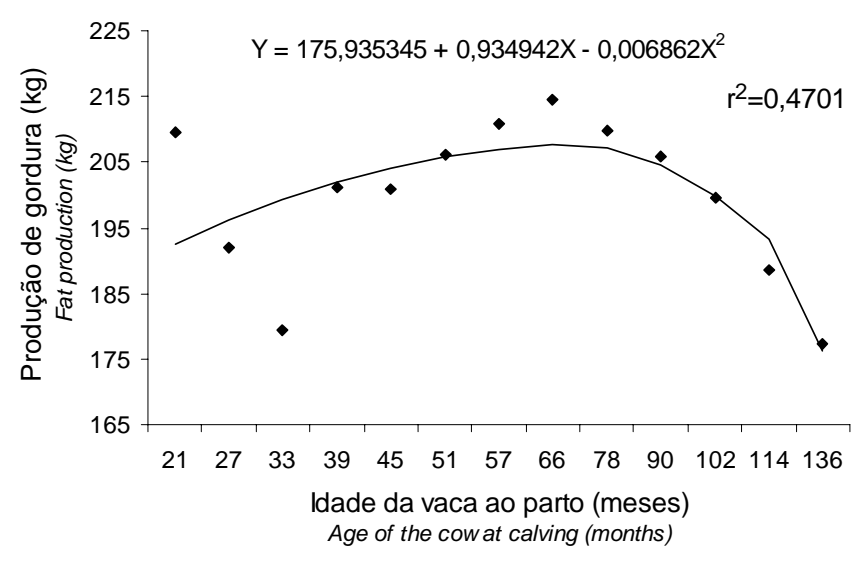

Figura 2 - Produção de gordura $(\mathrm{kg})$, segundo a idade da vaca ao parto.

Figure 2 - Fat production ( $\mathrm{kg}$ ), according to the age of the cow at calving.

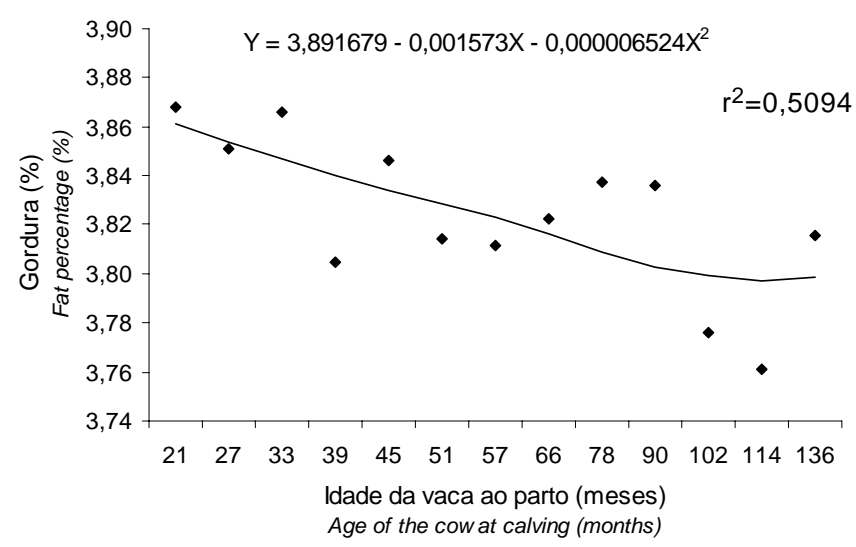

Figura 3 - Percentagem de gordura (\%), segundo a idade da vaca ao parto.

Figure 3 - Fat percentage (\%), according to the age of the cow at calving.
Tabela 4.

As médias de produção de leite e de gordura encontradas neste estudo foram superiores às encontradas em outros trabalhos realizados com animais da raça Pardo-Suíça no Brasil. Almeida et al. (1999), trabalhando com 2512 lactações de vacas PardoSuíças no Estado do Paraná, e Coelho (1990), com 864 lactações em um rebanho no Estado de São Paulo, encontraram médias de produção, desviospadrão e coeficientes de variação de $5428 \pm 2081 \mathrm{~kg}$ e $38,33 \%$ e $5642,66 \pm 37,39 \mathrm{~kg}$ e $20,51 \%$ de leite; e para produção de gordura $197,0 \pm 77 \mathrm{~kg}$ e $39,08 \%$ e $201,17 \pm 1,30 \mathrm{~kg}$ e $19,82 \%$, respectivamente.

As médias ajustadas, desvios-padrão e coeficientes de variação da percentagem de gordura, obtidos neste estudo, são semelhantes ao trabalho de Coelho (1990), que obteve 3,60 $\pm 0,01$ e 8,60\%. Entretanto, estas médias são superiores as encontradas por Almeida et al. (1999), de 3,37 $\pm 0,20$ e 5,93\%, ambos trabalhando com animais da raça Pardo-Suíça.

Os dados utilizados neste estudo são provenientes de grande número de rebanhos, constituindo o arquivo nacional de dados de produção de animais da raça Pardo-Suíça, ou seja, estes resultados representam a média nacional desta raça ao longo do período estudado. Desta forma pode-se justificar as diferenças de produção verificadas em relação aos estudos

Tabela 3 - Fatores de correção multiplicativos da produção de leite e gordura (2x, 305 dias), para a produção na idade adulta

Table 3 - Multiplicative adjustment factors of the production of milk and fat (2x, 305 days), for mature equivalent production

\begin{tabular}{lcc}
\hline $\begin{array}{l}\text { Classes de idades (meses) } \\
\text { Age class (months) }\end{array}$ & \multicolumn{2}{c}{$\begin{array}{c}\text { Fatores de correção } \\
\text { Adjustment factors }\end{array}$} \\
\cline { 2 - 3 } & $\begin{array}{c}\text { Produção } \\
\text { de leite } \\
\text { Milk } \\
\text { production }\end{array}$ & $\begin{array}{c}\text { Produção } \\
\text { de gordura } \\
\text { Fat } \\
\text { production }\end{array}$ \\
\hline $18-23$ & 1,307094 & 1,230636 \\
$24-29$ & 1,259812 & 1,232484 \\
$30-35$ & 1,219180 & 1,201982 \\
$36-41$ & 1,120063 & 1,113825 \\
$42-47$ & 1,087755 & 1,080656 \\
$48-53$ & 1,061122 & 1,058933 \\
$54-59$ & 1,018668 & 1,017287 \\
$60-71$ & 0,997868 & 1 \\
$72-83$ & 1 & 0,999908 \\
$84-95$ & 0,995937 & 0,999909 \\
$96-107$ & 1,017184 & 1,028070 \\
$108-119$ & 1,038071 & 1,059110 \\
$120-197$ & 1,102623 & 1,114490 \\
\hline
\end{tabular}

\section{R. Bras. Zootec., v.31, n.5, p.2043-2054, 2002}


Tabela 4 - Resumo da análise de variância da produção de leite, de gordura e percentagem de gordura, ajustadas para duas ordenhas (2x), 305 dias e idade adulta (ME)

Table 4 - Summary of analysis of variance for milk production, fat production and fat porcentage, adjustment for two milking daily, 305 days of lactation period and mature age

\begin{tabular}{|c|c|c|c|c|}
\hline \multirow[t]{2}{*}{$\begin{array}{l}\text { Fonte de variação } \\
\text { Source of variation }\end{array}$} & \multirow[t]{2}{*}{$\begin{array}{l}\mathrm{G} \\
D F\end{array}$} & \multicolumn{3}{|c|}{$\begin{array}{l}\text { Quadrado médio } \\
\text { Mean square }\end{array}$} \\
\hline & & $\begin{array}{l}\text { Produção de leite } \\
\text { Milk production } \\
\text { (P-value) }\end{array}$ & $\begin{array}{l}\text { Produção de gordura } \\
\text { Fat production } \\
\text { (P-value) }\end{array}$ & $\begin{array}{l}\text { Percentagem de gordura } \\
\text { Fat percentage } \\
\text { (P-value) }\end{array}$ \\
\hline $\begin{array}{l}\text { Rebanho } \\
\text { Herd }\end{array}$ & 200 & $38.992 .203,86(0,0001)$ & $44.305,68(0,0001)$ & $1,52(0,0001)$ \\
\hline $\begin{array}{l}\text { Ano de parto } \\
\text { Year of calving }\end{array}$ & 19 & $121.567 .840,85(0,0001)$ & $166.950,10(0,0001)$ & $2,00(0,0001)$ \\
\hline $\begin{array}{l}\text { Época de parto } \\
\text { Season of calving }\end{array}$ & 3 & $10.377 .651,09(0,0001)$ & $9.781,490(0,0045)$ & $0,21(0,1419)$ \\
\hline $\begin{array}{l}\text { Interação ano } \mathrm{x} \text { época de parto } \\
\text { Interaction year and season of calving }\end{array}$ & 57 & $2.649 .552,59(0,0002)$ & $3.685,91(0,0017)$ & $0,20(0,0012)$ \\
\hline $\begin{array}{l}\text { Grupo genético } \\
\text { Genetic group }\end{array}$ & 2 & $69.987 .101,79(0,0001)$ & $128.534,77(0,0001)$ & $0,74(0,0020)$ \\
\hline
\end{tabular}

descritos acima, já que estes foram realizados em regiões específicas ou dentro de poucos rebanhos, não representando a média nacional.

As médias das características produtivas estudadas, produção de leite, de gordura e da percentagem de gordura, de acordo com os anos e épocas de parição, encontram-se nas Tabelas 5, 6 e 7 .

Neste estudo foi verificado que existe efeito significativo $(\mathrm{P}<0,01)$ da interação ano-época de

Tabela 5 - Médias estimadas (kg) e desvios-padrão (DP) da produção de leite segundo o ano e épocas de partos Table 5 - Estimate averages and standard deviation of milk production by year and season of calving

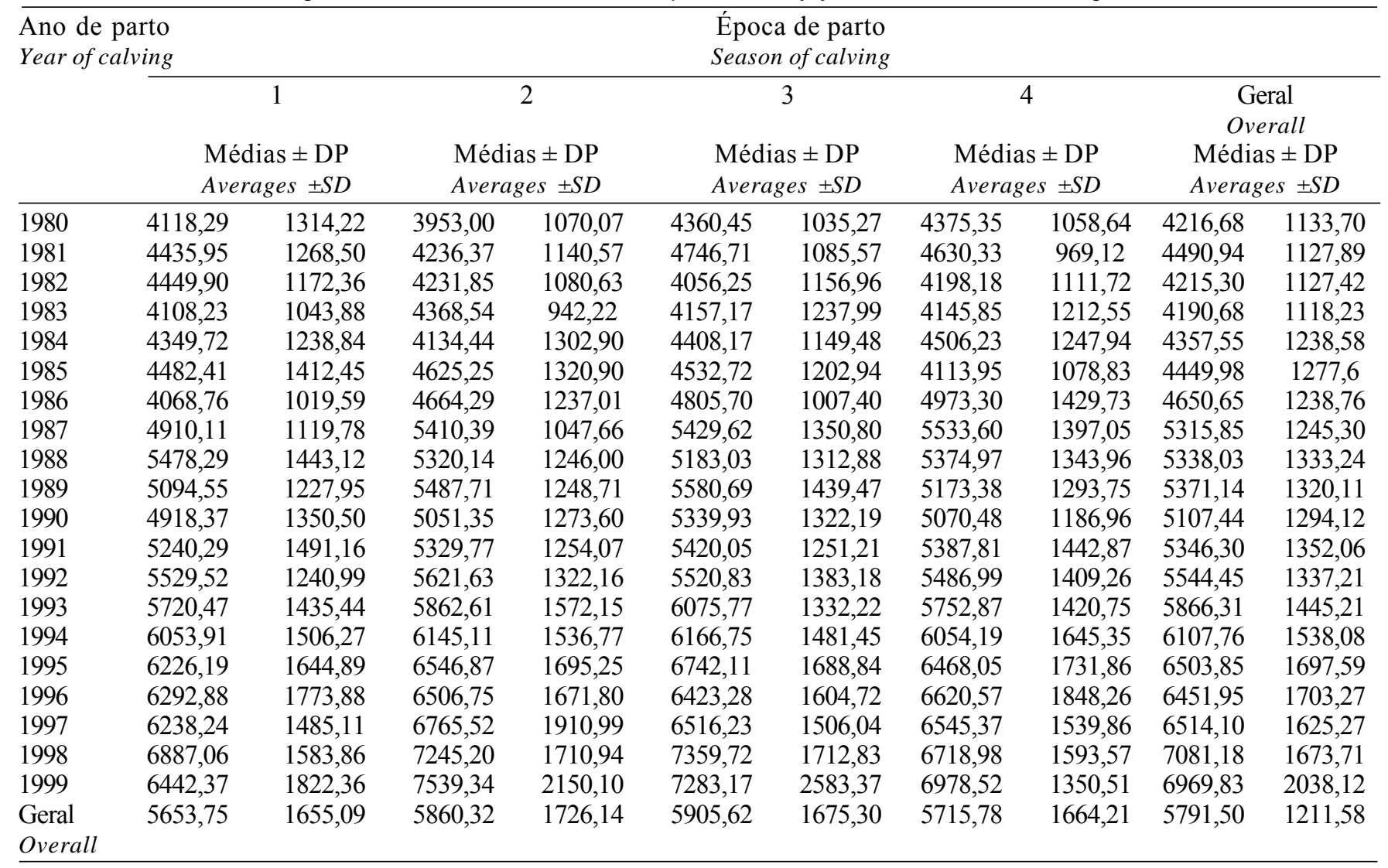

\section{R. Bras. Zootec., v.31, n.5, p.2043-2054, 2002}


Tabela 6 - Médias estimadas (kg) e desvios-padrão (DP) da produção de gordura segundo o ano e épocas de partos Table 6 - Estimate averages and standard deviation of fat production by year and season of calving

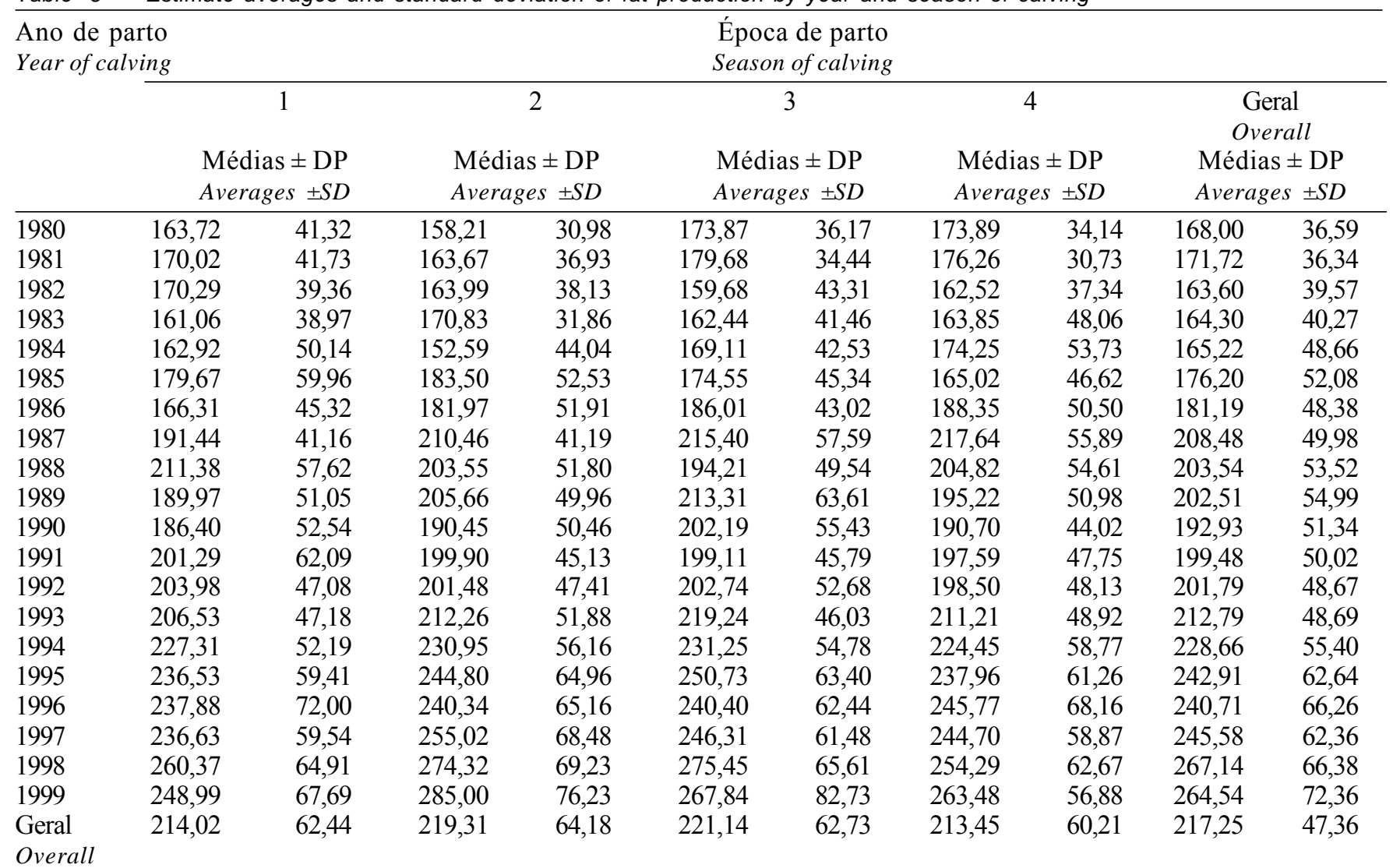

partos com as características produtivas estudadas; produção de leite, de gordura e percentagem de gordura. No estudo de Nobre (1983), utilizando as produções do rebanho leiteiro da Universidade Federal de Viçosa, foi observado o efeito da interação ano-época de parto. Também, de forma semelhante, Freitas (1988) e Matos et al. (1997), verificaram efeito $(\mathrm{P}<0,01)$ da interação ano-época de partos sobre a produção de leite e de gordura.

$\mathrm{O}$ ano de parto influi sobre a produção de leite, de gordura e da percentagem de gordura, respondendo por 7,27; 7,23 e 2,20\% das somas de quadrados totais, respectivamente. As médias de produção de leite e gordura aumentaram gradativamente a partir de 1980, onde a produção era de $4216,68 \mathrm{~kg}$ de leite e 168,00 $\mathrm{kg}$ de gordura, passando a ser de $7081,18 \mathrm{~kg}$ de leite e 264,54 kg de gordura em 1998.

A percentagem de gordura apresenta oscilação de suas médias estimadas do período de 1980 a 1988 , onde a partir de 1989 até 1999 está média tem comportamento relativamente estável, variando de 3,65 a $3,80 \%$. Estes resultados são extremamente importantes para o desempenho da raça, já que dificilmente consegue-se obter aumentos de produção de leite sem que ocorra diminuições no teor de gordura, devido à correlação negativa entre estas características. Diversos autores, trabalhando com raças especializadas na produção de leite em diversas localidades no Brasil têm verificado efeitos significativos do ano de parto sobre a produção de leite, gordura e percentagem de gordura (Matos et al., 1997; Pimpão et al., 1997; Ribas et al., 1999).

A época de parto influenciou as produções de leite e de gordura, entretanto a percentagem de gordura não foi influenciada por esta variável. As lactações iniciadas nos meses de outono e inverno (de abril a setembro) apresentaram as maiores produções de leite e de gordura. A percentagem de gordura, no entanto, permaneceu inalterada ao longo das épocas de parição. As diferenças entre as épocas de maior e menor produção (inverno ou época 3, e verão ou época 1), apresentam uma vantagem de $251,87 \mathrm{~kg}$ de leite e $7,12 \mathrm{~kg}$ de gordura para a época 3 .

Outros autores têm encontrado resultados semelhantes aos deste estudo. Almeida et al. (1999), trabalhando com vacas da raça Pardo-Suíça, encon- 
Tabela 7 - Médias estimadas e desvios-padrão (DP) da percentagem de gordura segundo o ano e épocas de partos Table 7 - Estimate averages and standard deviation of fat percentage by year and season of calving

\begin{tabular}{|c|c|c|c|c|c|c|c|c|c|c|}
\hline \multicolumn{5}{|c|}{$\begin{array}{l}\text { Ano de parto } \\
\text { Year of calving }\end{array}$} & \multicolumn{4}{|c|}{$\begin{array}{l}\text { Época de parto } \\
\text { Season of calving }\end{array}$} & & \\
\hline & \multicolumn{2}{|c|}{1} & \multicolumn{2}{|c|}{2} & \multicolumn{2}{|c|}{3} & $\begin{array}{l}\text { Méc } \\
\text { Aver }\end{array}$ & 4 & $\begin{array}{c}\text { Geral } \\
\text { Overall }\end{array}$ & $\begin{array}{l}\text { Médias } \pm \mathrm{DP} \\
\text { Averages } \pm S D\end{array}$ \\
\hline 1980 & 4,08 & 0,62 & 4,11 & 0,61 & 4,04 & 0,49 & 4,04 & 0,54 & 4,06 & 0,56 \\
\hline 1981 & 3,89 & 0,36 & 3,91 & 0,33 & 3,81 & 0,20 & 3,83 & 0,28 & 3,86 & 0,30 \\
\hline 1982 & 3,86 & 0,26 & 3,91 & 0,33 & 3,96 & 0,26 & 3,92 & 0,32 & 3,92 & 0,30 \\
\hline 1983 & 3,95 & 0,41 & 3,94 & 0,34 & 3,96 & 0,36 & 3,98 & 0,34 & 3,96 & 0,36 \\
\hline 1984 & 3,75 & 0,32 & 3,73 & 0,32 & 3,86 & 0,30 & 3,86 & 0,36 & 3,80 & 0,33 \\
\hline 1985 & 4,00 & 0,32 & 3,96 & 0,35 & 3,86 & 0,31 & 4,01 & 0,34 & 3,96 & 0,33 \\
\hline 1986 & 4,07 & 0,39 & 3,90 & 0,34 & 3,87 & 0,30 & 3,83 & 0,33 & 3,91 & 0,35 \\
\hline 1987 & 3,91 & 0,28 & 3,89 & 0,21 & 3,96 & 0,28 & 3,94 & 0,27 & 3,93 & 0,26 \\
\hline 1988 & 3,86 & 0,32 & 3,83 & 0,32 & 3,76 & 0,27 & 3,80 & 0,31 & 3,82 & 0,31 \\
\hline 1989 & 3,72 & 0,36 & 3,74 & 0,29 & 3,81 & 0,43 & 3,78 & 0,37 & 3,76 & 0,36 \\
\hline 1990 & 3,80 & 0,38 & 3,77 & 0,35 & 3,78 & 0,36 & 3,78 & 0,31 & 3,78 & 0,35 \\
\hline 1991 & 3,84 & 0,32 & 3,77 & 0,33 & 3,69 & 0,32 & 3,70 & 0,33 & 3,75 & 0,33 \\
\hline 1992 & 3,69 & 0,36 & 3,61 & 0,45 & 3,68 & 0,37 & 3,64 & 0,35 & 3,65 & 0,39 \\
\hline 1993 & 3,64 & 0,39 & 3,66 & 0,41 & 3,63 & 0,41 & 3,70 & 0,37 & 3,66 & 0,40 \\
\hline 1994 & 3,80 & 0,50 & 3,78 & 0,40 & 3,77 & 0,38 & 3,74 & 0,42 & 3,77 & 0,43 \\
\hline 1995 & 3,83 & 0,38 & 3,76 & 0,37 & 3,73 & 0,36 & 3,71 & 0,43 & 3,76 & 0,39 \\
\hline 1996 & 3,77 & 0,37 & 3,70 & 0,40 & 3,74 & 0,39 & 3,73 & 0,41 & 3,74 & 0,39 \\
\hline 1997 & 3,80 & 0,39 & 3,79 & 0,36 & 3,77 & 0,36 & 3,75 & 0,37 & 3,78 & 0,37 \\
\hline 1998 & 3,78 & 0,36 & 3,79 & 0,39 & 3,75 & 0,36 & 3,79 & 0,42 & 3,78 & 0,38 \\
\hline 1999 & 3,90 & 0,45 & 3,82 & 0,48 & 3,77 & 0,53 & 3,79 & 0,41 & 3,84 & 0,46 \\
\hline $\begin{array}{l}\text { Geral } \\
\text { Overall }\end{array}$ & 3,81 & 0,40 & 3,76 & 0,39 & 3,76 & 0,38 & 3,76 & 0,39 & 3,78 & 0,34 \\
\hline
\end{tabular}

traram efeito significativo da época de parto sobre a produção de leite e de gordura. Entretanto, para a porcentagem de gordura, a época de parto não apresentou efeito significativo. Resultados semelhantes obteve Nobre (1983), Ray et al. (1992), Norman et al. (1995) e Balieiro (1997).

O efeito de rebanho foi importante fonte de variação $(\mathrm{P}<0,01)$, respondendo por 24,$54 ; 20,20 \mathrm{e}$ $17,63 \%$ das somas de quadrados totais da produção de leite, de gordura e da percentagem de gordura, respectivamente. Estes resultados estão de acordo com Ribas et al. (1996) e Pimpão et al. (1997), trabalhando com vacas da raça Holandesa, com 281 e 36 rebanhos respectivamente, e ainda com o estudo de Almeida et al. (1999), que, trabalhando com vacas Pardo-Suíças em 38 rebanhos, observaram efeito desta variável sobre a produção de leite, de gordura e da percentagem de gordura.

Houve diferença $(\mathrm{P}<0,01)$ na produção de leite, de gordura e da percentagem de gordura de acordo com os grupos genéticos. As médias ajustadas e os respectivos desvios-padrão podem ser observados na Tabela 8 .
Os animais do grupo genético puro de origem importada (POI), representados por animais nascidos e criados, em sua maioria, nos Estados Unidos, sendo posteriormente importados para o Brasil, apresentaram as maiores quantidades de leite e gordura produzidos do que animais do grupo genético puro de origem (PO), representado por animais nascidos no país, estes por sua vez, tiveram maiores produções de leite e gordura do que o grupo genético puro por cruza (PC), composto também por animais nascidos no Brasil.

A percentagem de gordura foi semelhante nos animais dos grupos genéticos POI e PO, entretanto, estes resultados foram superiores aos valores obtidos com os animais do grupo genético PC. RIBAS et al. (1996) e PIMPÃO et al. (1997), com vacas Holandesas, e RIBAS et al. (1999), com animais da raça Jersey, também verificaram a influência do grupo genético sobre a produção de leite, de gordura e da porcentagem de gordura.

Parâmetros genéticos

As médias observadas, os desvios-padrão, os coeficientes de variação e o número de lactações, 
Tabela 8 - Número de observações (N), médias estimadas e respectivos desvios-padrão (DP) da produção de leite, de gordura e percentagem de gordura, ajustadas para duas ordenhas (2x), 305 dias de período de lactação e idade adulta (ME), segundo o grupo genético

Table 8 - Number of observations, estimate averages and respective standard deviation of the production of milk, fat and fat percentage, adjusted for two milking daily (2x), 305 days of lactation period and adult age, according to the genetic group

\begin{tabular}{|c|c|c|c|c|c|c|c|}
\hline \multirow[t]{2}{*}{$\begin{array}{l}\text { Grupo genético } \\
\text { Genetic group }\end{array}$} & \multirow[t]{2}{*}{$\begin{array}{l}\mathrm{N} \\
N\end{array}$} & \multicolumn{2}{|c|}{$\begin{array}{l}\text { Produção de leite } \\
\text { Milk production }\end{array}$} & \multicolumn{2}{|c|}{$\begin{array}{l}\text { Produção de gordura } \\
\text { Fatproduction }\end{array}$} & \multicolumn{2}{|c|}{$\begin{array}{c}\text { Percentagem de gordura } \\
\text { Fatpercentage }\end{array}$} \\
\hline & & $\begin{array}{l}\text { Médias } \\
\text { Averages } \\
\text { deviation }\end{array}$ & $\begin{array}{c}\text { DP } \\
\text { Standard } \\
\text { deviation }\end{array}$ & $\begin{array}{l}\text { Médias } \\
\text { Averages } \\
\text { deviation }\end{array}$ & $\begin{array}{c}\mathrm{DP} \\
\text { Standard } \\
\text { deviation }\end{array}$ & $\begin{array}{l}\text { Médias } \\
\text { Averages } \\
\text { deviation }\end{array}$ & $\begin{array}{c}\mathrm{DP} \\
\text { Standard } \\
\text { deviation }\end{array}$ \\
\hline POI & 2385 & $6160,32^{a}$ & 1583,97 & $233,07^{\mathrm{a}}$ & 63,08 & $3,79^{a}$ & 0,41 \\
\hline $\mathrm{PO}$ & 7037 & $5711,31^{b}$ & 1637,03 & $215,62^{b}$ & 62,17 & $3,79^{\mathrm{a}}$ & 0,36 \\
\hline $\mathrm{PC}$ & 1767 & $5613,01^{\mathrm{c}}$ & 1919,73 & $202,38^{c}$ & 59,03 & $3,69^{b}$ & 0,46 \\
\hline $\begin{array}{l}\text { Geral } \\
\text { Overall }\end{array}$ & 11189 & 5791,50 & 1211,58 & 217,25 & 47,36 & 3,77 & 0,34 \\
\hline
\end{tabular}

Médias seguidas de letras iguais não diferem entre si, ao nível de $5 \%$ de probabilidade, pelo teste de Tukey.

Values within column with different letters significantly differ $(P<0.05)$ by Tukey test.

para a produção de leite e de gordura ajustadas para duas ordenhas, 305 dias de lactação e à idade adulta, são apresentados na Tabela 9. Estas médias de produção se referem aos animais utilizados para a avaliação genética da raça Pardo-Suíça em função de diferentes restrições utilizadas na obtenção dos arquivos analisados.

Os componentes de variância, para a produção de leite e gordura, estimados pelo modelo estatístico utilizado, podem ser observados na Tabela 10. Os parâmetros genéticos estimados neste estudo, coefi-

Tabela 9 - Médias, desvios-padrão (D.P.) e coeficientes de variação (C.V.) para produção de leite e gordura, ajustada para duas ordenhas diárias, 305 dias de lactação e à idade adulta

Table 9 - Averages, standard deviation (D.P.) and variation coefficients (C.V.) of the milk and fat yield, adjusted for two milking daily, 305 days of lactation and adult age

\begin{tabular}{lcc}
\hline $\begin{array}{l}\text { Parâmetros } \\
\text { Parameters }\end{array}$ & \multicolumn{2}{c}{$\begin{array}{c}\text { Características } \\
\text { Characteristics }\end{array}$} \\
\hline & $\begin{array}{c}\text { Produção de leite } \\
\text { Milkyield }\end{array}$ & $\begin{array}{c}\text { Produção de gordura } \\
\text { Fatyield }\end{array}$ \\
\hline Média $(\mathrm{kg})$ & 5879,85 & 221,03 \\
Average $(\mathrm{kg})$ & & \\
D.P. $(\mathrm{kg})$ & 1655,79 & 62,52 \\
C.V.(\%) & 28,16 & 28,29 \\
\hline
\end{tabular}

cientes de herdabilidade, de repetibilidade e correlação genética entre as características produtivas, estão apresentados na Tabela 11.

Os valores dos coeficientes de herdabilidade e repetibilidade estimados estão de acordo com aqueles obtidos por autores que utilizaram metodologias semelhantes para a estimativa de parâmetros genéticos, como os trabalhos de Almeida et al. (1997), Freitas et al. (1999a), Freitas et al. (1999b), e Araújo (2000). As correlações genéticas estimadas neste estudo são semelhantes aos valores obtidos nos tra-

Tabela 10 - Componentes de variância genética aditiva $\left(\hat{\sigma}_{\mathrm{a}}^{2}\right)$, ambiente permanente $\left(\hat{\sigma}_{\mathrm{C}^{1}}^{2}\right)$, e residual $\left(\hat{\sigma}_{\mathrm{e}}^{2}\right)$ para a produção de leite e gordura ajustada a duas ordenhas, 305 dias de lactação e à idade adulta

Table 10 - Components of addictive genetic variance $\left(\hat{\sigma}_{\mathrm{a}}^{2}\right)$, environment permanent $\left(\hat{\sigma}_{\mathrm{C}^{1}}^{2}\right)$, and residual $\left(\hat{\sigma}_{\mathrm{e}}^{2}\right)$ for milk and fat yield adjusted for two milking daily, 305 days of lactation and adult age

\begin{tabular}{lcc}
\hline Parâmetros & \multicolumn{2}{c}{ Características } \\
& \multicolumn{2}{c}{ Characteristics } \\
\cline { 2 - 3 } & $\begin{array}{c}\text { Produção de leite } \\
\text { Milkyield }\end{array}$ & $\begin{array}{c}\text { Produção de gordura } \\
\text { Fat yield }\end{array}$ \\
\hline$\hat{\sigma}_{\mathrm{a}}^{2}$ & 628681,231167 & 940,956619 \\
$\hat{\sigma}_{\mathrm{c}^{1}}^{2}$ & 47751,616635 & 22,230542 \\
$\hat{\sigma}_{\mathrm{e}}^{2}$ & 1030642,695464 & 1682,579371 \\
\hline
\end{tabular}


Tabela 11 - Estimativas de herdabilidade, repetibilidade, proporções da variância fenotípica em relação aos efeitos permanentes de meio ( ), efeito residual (e) e correlação genética da produção de leite e gordura ajustada para duas ordenhas diárias, 305 dias de lactação e à idade adulta

Table 11 - Estimates of heritability, repeatability, proportions of the fenotipic variance in relation to the permanent effects of environmental ( ), residual effect (e) and genetic correlation among the studied productive characteristics

\begin{tabular}{lcc}
\hline $\begin{array}{l}\text { Parâmetros } \\
\text { Parameters }\end{array}$ & \multicolumn{2}{c}{ Características } \\
\cline { 2 - 3 } & $\begin{array}{c}\text { Produção de leite } \\
\text { Milkyield }\end{array}$ & $\begin{array}{c}\text { Produção de gordura } \\
\text { Fatyield }\end{array}$ \\
\hline $\begin{array}{l}\text { Herdabilidade } \\
\text { Heritability }\end{array}$ & 0,37 & 0,36 \\
$\begin{array}{l}\text { Repetibilidade } \\
\text { Repeatability }\end{array}$ & 0,40 & 0,37 \\
$\quad \mathrm{C}^{1}$ & 0,03 & 0,01 \\
$\quad$ e & 0,60 & 0,64 \\
$\begin{array}{l}\text { Correlaçãogenética } \\
\text { Genetic correlation }\end{array}$ & 0,96 & \\
\hline
\end{tabular}

balhos de Matos et al. (1997), Neves et al. (1998) e Freitas et al. (1999a).

\section{Conclusões}

Os resultados obtidos revelam a necessidade do ajustamento para as produções de leite e gordura para os efeitos do número de ordenhas diárias, período de lactação e idade da vaca ao parto quando o objetivo é a avaliação do potencial produtivo dos animais desta raça.

As médias de produção de leite, gordura e percentagem de gordura apresentadas neste estudo demonstram que os animais da raça Pardo-Suíça apresentam elevado desempenho produtivo nos rebanhos brasileiros. As variáveis ano de parto, época de parto, ano-época de parto, rebanho e grupo genético interferiram de forma significativa sobre a produção dos animais desta raça.

As estimativas de herdabilidade demonstram a possibilidade de modificações quantitativas para se elevar à média das produções de leite e gordura por meio de seleção individual de vacas. As estimativas de repetibilidade obtidas para as características estudadas, produção de leite e de gordura, indicam que existe possibilidade de uma única lactação da vaca ser utilizada no processo de seleção.

A alta correlação entre a produção de leite e de gordura, obtida neste estudo em animais da raça Pardo-Suíça, sugere que os genes que atuam na produção de leite também respondem pela produção de gordura, de tal forma que a seleção para a produção de leite resulta, indiretamente, em aumentos na produção de gordura.

\section{Literatura Citada}

ALMEIDA, R.; RIBAS, N.P.; MARCONDES, E.A. Estudo de alguns fatores do meio ambiente sobre as produções de leite, gordura e proteína em vacas da raça Pardo-Suíça no Estado do Paraná. In: REUNIÃO ANUAL DA SOCIEDADE BRASILEIRA DE ZOOTECNIA, 36., 1999, Porto Alegre. Anais... Porto Alegre: Sociedade Brasileira de Zootecnia, 1999. p.159.

ALMEIDA, R.; RIBAS, N.P.; MONARDES, H.G. Estudo dos efeitos de meio ambiente sobre as características produtivas de vacas da raça Holandesa na região da Batavo, Paraná. In: REUNIÃO ANUAL DA SOCIEDADE BRASILEIRA DE ZOOTECNIA, 34, 1997, Juiz de Fora. Anais... Juiz de Fora: Sociedade Brasileira de Zootecnia, 1997. p.65.

AMOS, H.E.; TERRY, K.; LOEWENSTEIN, M. Influence of milking frequency on productive and reproductive efficiencies of dairy cows. Journal of Dairy Science, v.68, n.3, p.732$739,1985$.

ARAÚJO, C.V. Efeito da interação reprodutor x rebanho sobre a produção de leite na raça Holandesa. Viçosa, MG: Universidade Federal de Viçosa, 2000. 80p. Dissertação (Mestrado em Zootecnia) - Universidade Federal de Viçosa, 2000.

BALIEIRO, J.C.C. Aspectos genéticos e fenotípicos em características produtivas e reprodutivas do rebanho leiteiro da Universidade Federal de Viçosa. Viçosa, MG: Universidade Federal de Viçosa, 1997. 109p. Dissertação (Mestrado em Zootecnia) - Universidade Federal de Viçosa, 1997.

BARBOSA, S.B.P.; MANSO, H.C.; SILVA, L.O.C. Estudo do período de lactação em vacas Holandesas no Estado de Pernambuco. Revista da Sociedade Brasileira de Zootecnia, v.23, n.3, p.465-475, 1994.

BOLDMAN, K.G.; KRIESE, L.A.; Van VLECK, L.D. et al. A manual for use of MTDFREML: A set of programs to obtain estimates of variances and covariances. Lincoln: Department of Agriculture/Agricultural Research Service, 1995. 120p.

CAMPOS, J.M. Aspectos reprodutivos e produtivos em um sistema de produção de leite, na microrregião de Viçosa, Estado de Minas Gerais. Viçosa, MG: Universidade Federal de Viçosa, 1987. 109p. Dissertação (Mestrado em Zootecnia) - Universidade Federal de Viçosa, 1987.

COELHO, M.M. Fatores de meio e genéticos em características produtivas e reprodutivas nas raças Holandesa e Pardo-Suíça. Lavras, MG: Universidade Federal de Lavras, 1990. 118p. Dissertação (Mestrado em Zootecnia) - Universidade Federal de Lavras, 1990.

CONCEIÇÃO Jr., V.; SILVA, H.M.; PEREIRA, C.S. Fatores ambientes e genéticos que afetam a produção de leite e gordura em vacas da raça Holandesa. Arquivos Brasileiros de Medicina Veterinária e Zootecnia, v.45, n. 1, p.81-88, 1993.

COSTA, C.N. Genetic relationship for milk and fat yields between Brazilian and United States Holstein cattle populations. New York: Cornell University, 1998. 175p. Tesis (Doctor of Philosophy) - Cornell University, 1998.

R. Bras. Zootec., v.31, n.5, p.2043-2054, 2002 
DePETERS, E.J.; SMITH, N.E.; ACEDO-RICO, J. Three or two times daily milking of older cows and first lactation cows for entire lactations. Journal of Dairy Science, v.68, n.1, p.123-132, 1985.

FREEMAN, A.E. Age adjustment of production records: history and basic problems. Journal of Dairy Science, v.56, p.941-946, 1973.

FREITAS, A.F. Fatores genéticos e de meio em algumas características produtivas de um rebanho leiteiro mestiço. Viçosa, MG: Universidade Federal de Viçosa, 1988. 117 p. Tese (Doutorado em Zootecnia) - Universidade Federal de Viçosa, 1988.

FREITAS, A.F.; DURÃES, M.C.; TEIXEIRA, N.M. et al. Estimativas de parâmetros genéticos para produção de leite e gordura de vacas Holandesas-PO no Estado de Minas Gerais. In: REUNIÃO ANUAL DA SOCIEDADE BRASILEIRA DE ZOOTECNIA, 36., 1999, Porto Alegre. Anais... Porto Alegre: Sociedade Brasileira de Zootecnia, 1999a. p. 150 .

FREITAS, A.F.; TEIXEIRA, N.M.; DURÃES, M.C. et al. Estimativas de parâmetros genéticos para produções de leite, gordura e proteína de vacas mestiças. In: REUNIÃO ANUAL DA SOCIEDADE BRASILEIRA DE ZOOTECNIA, 36., 1999, Porto Alegre. Anais... Porto Alegre: Sociedade Brasileira de Zootecnia, 1999b. p.151.

HOURI NETO, M. Interação genótipo-ambiente e avaliação genética de reprodutores da raça Holandesa, usados no Brasil e nos Estados Unidos da América. Belo Horizonte: Universidade Federal de Minas Gerais, 1996. 204p. Tese (Doutorado em Melhoramento Animal) - Universidade Federal de Minas Gerais, 1996.

KAISER, F.R. Pardo-Suíço - Consolidando espaços no leite. DBO Rural, n.215, p.1-2, 1998.

MARTINEZ, M.L.; LEE, A.J.; LIN, C.Y. Multiplicative ageseason adjustments factors by maximum likelihood, gross comparisons, and paired comparisons. Journal of Dairy Science, v.73, n.3, p.819-825, 1990.

MATOS, R.S.; RORATO, P.R.N.; FERREIRA, G.B. et al. Estudo dos efeitos genéticos e de meio ambiente sobre a produção de leite e gordura da raça Holandês no Estado do Rio Grande do Sul. Ciência Rural, v.27, n.3, p.465-471, 1997.

McDOWELL, R.E.; CAMOENS, J.K.; Van VLECK, L.D. et al. Factors affecting performance of Holstein in subtropical regions of México. Journal of Dairy Science, v.59, n.4, p.722-729, 1976.

NEVES, C.J.; FERREIRA, G.B.; FERNANDES, H.D. et al. Estimativas de parâmetros genéticos para a produção de leite e gordura em bovinos da raça Holandesa no estado de Goiás. In: REUNIÃO ANUAL DA SOCIEDADE BRASILEIRA DE ZOOTECNIA, 35., 1998, Botucatu. Anais... Botucatu: Sociedade Brasileira de Zootecnia, 1998. p.103.

NOBRE, P.R.C. Fatores genéticos e de meio em características produtivas e reprodutivas do rebanho leiteiro da Universidade Federal de Viçosa, Estado de Minas Ge- rais. Viçosa, MG: Universidade Federal de Viçosa, 1983. 128p. Dissertação (Mestrado em Zootecnia) - Universidade Federal de Viçosa, 1983.

NORMAN, H.D.; MEINERT, T.R.; SCHUTZ, M.M. et al. Age and seasonal effects on Holstein yield for four regions of the United States over time. Journal of Dairy Science, v.78, n.8, p.1855-1861, 1995.

PIMPÃO, C.T.; RIBAS, N.P.; MONARDES, H.G. et al. Estudo dos efeitos de meio ambiente sobre as características produtivas de vacas da raça Holandesa da região de Arapoti, Estado do Paraná. Revista Brasileira de Zootecnia, v.26, n.3, p.494-500, 1997.

RAY, D.E.; HALBACH, T.J.; ARMSTRONG, D.V. Season and lactation number effects on milk production and reproduction of dairy cattle in Arizona. Journal of Dairy Science, v.75, n.11, p.2976-2983, 1992.

RENNÓ, F.P. A raça Pardo-Suíça. In: MINAS LEITE, 2., 2000, Juiz de Fora. Anais... Juiz de Fora: EMBRAPA, 2000. p.53.

RIBAS, N.P. Fatores de meio e genéticos em características produtivas e reprodutivas de rebanhos Holandês da bacia leiteira de Castrolanda, Estado do Paraná. Viçosa, MG: Universidade Federal de Viçosa, 1981. 141p. Dissertação (Mestrado em Zootecnia) - Universidade Federal de Viçosa, 1981.

RIBAS, N.P.; ALMEIDA, R.; MARCONDES, E.A. Estudo de alguns fatores de meio sobre as produções de leite, gordura $\mathrm{e}$ proteína em vacas da raça Jersey no Estado do Paraná. In: REUNIÃO ANUAL DA SOCIEDADE BRASILEIRA DE ZOOTECNIA, 36., 1999, Porto Alegre. Anais... Porto Alegre: Sociedade Brasileira de Zootecnia, 1999. p.159.

RIBAS, N.P.; MONARDES, H.G.; MOLENTO, C.F.M. et al. Estudo dos efeitos de meio ambiente sobre as características produtivas de vacas da raça Holandesa no Estado do Paraná. In: REUNIÃO ANUAL DA SOCIEDADE BRASILEIRA DE ZOOTECNIA, 33., 1996, Fortaleza. Anais... Fortaleza: Sociedade Brasileira de Zootecnia, 1996. p.9.

SAS INSTITUTE. SAS System for Linear Models. 3.ed. Cary: 1991.

TORRES, R.A. Efeito da heterogeneidade de variância na avaliação genética de bovinos da raça Holandesa no Brasil. Belo Horizonte: Universidade Federal de Minas Gerais, 1998. 123p. Tese (Doutorado em Ciência Animal) Universidade Federal de Minas Gerais, 1998. 